\section{Ein Beitrag zur Genese der Mastzellen der Haut.')}

\section{Von Priv.-Doz. Dr. Julius Heller in Charlottenburg-Berlin.}

Seitdem Ehrlich die Hypothese aufgestellt hat, daß die Zellgranula als Stoffwechselprodukte der Zelle anzuseben sind und die Bestimmung haben, an die Umgebung abgegeben zu werden, hat die Frage nach der Genese der granulahaltigen Zellen eine erhöhte $\mathrm{Be}$ deutung gewonnen. Zweifellos bedarf im erkrankten Zustande ein Orgaı einer verstärkten Ernährung zur Erhaltung der den patholo girchen Prozeß zusammensetzenden Gewebselemente. Stammen nun die Grauula enthaltenden Zellen aus dem Organ selbst, so bleibt festzustellen, wie das letztere das zur Produktion der Granulazellen nötige Material an sich zieht; stammen die Granula enthaltenden Zellen aus dem Blute, so vereinfacht siclı der ganze Vorgano wesentlich. Von diesem Standpunkte ans gewinnt der 7.wischen der Unnaschen und Neisserschen Schule ausgefochtene Streit über die bämatogene oder listiogene Natur der Plasmazellen an Bedeutung. Zweifellos hat sich die größBere Zahl der Forscher ${ }^{2}$ ) für die hämatogene Natur der Plasmazellen entschieden und damit eine Stiitze für den Ehrlich und Lazarıs. schell Satz gegeben. daß „die Granula im allgemeinen bei allen Tierspezies nur in den Zellen des Blıtes enthalten sind, welche zur Answanderung bestimmt und befähigt sind." Weit weniger geklärt als die Anffassungen über die Genese der Plasmazellen sind unsere Anschauungen über die Entstehung der Mastzellen. Es sei ganz kurz gestattet, den Stand der Mastzellenfrage hier zu skizzieren.

Wir verstehen bekanntlich inter Mastzellen die zuerst 1877 von Ehrlich beschriebenen Zellen, welche bei Tinktion mit basisclien Anilinfarbstoffen zablreiche Körnchen zeigen, welche wegen ihrer Verwandtschaft zu den basischen Anilinstoffen als basophile Granula bez.cichnet werden. Diese Granula haben die Eigentïmlichkeit, sich unter bestimmten äußeren Bedingungen metachromatisch zu färben, das heißt aus Farbgernischen bestimmte Farben an sich zu ziehen und siclı so im Gegensatz zu den übrigen Gewebselementen distinkt zu färbeı. Es laandelt sich stets um violette bis blaue Farbstoffe, die die Kerue blaıl, die Mastzellengranula rotviolett färben. Worauf diese Metacliromasie beruht, ist nicht ganz siclier. Meiner Leberzeugung nach ist das Vorhandensein von Mucin oder äbulichen Substanzen ein wichtiger Faktor für das Zustandekommen der Metachromasie.

Der Name „Mastzellen" vurde von Ehrlich gewählt, weil diese Zelleı besonders häufig im überernährten Gewebe, zum Beispiel bei chronisclien Entzündungen vorkommen; er wurde auch für die basophil granulierten Leukozyten beibehalten. Die Mastzellen des Blutes sind aber Leukozyten, welche gewöhnlich bei der Färbung mit Methylenblau und seinen Derivaten mehr oder weniger stark gefürbte Kerne und charakteristiscbe Granulationen zeigen. Sie kommen in geringer Zahl im normalen Blıte vor: nur $0,5 \%$ der Leukozyten sind Mastzellen. L. Michaelis hat nachgewiesen. dal3 die Granula der Blittmastzellen leicht wasserlöslicl sind. Demgegenüber sind die Mastzellen des Bindegewebes, speziell der Hant, zienlich große Gebilde, die eine entweder rundliche, längliche oder vielgestaltige Form zeigeıt. Sie haben nur einen Kern, der sich bei der Färbung nit Iethylenblaufarbstoff oder seinen Derivaten schwach blau färbt. Das ganze Protoplasma dieser Zellen ist bedeckt mit den Granulationen, welche bei entsprechender Tinktion die charakteristische metachromatische Färbung zeigen. Diese Mastzellen finden sich in der normalen Haut, sie kommen abèr außerordentlich zahlreich bei den meisten chronischen Entzündungen vor.

Das ganze Aussehen der Mastzellen der Haut und des Blutes ist ein so verschiedenartiges, dak man von vornherein geneigt ist, beide Zellenformen voneinander zu scheiden. Diese Scheidung erscheint um so einleuchtender, wenn man sich daran erinnert, dab in allen den jenigen Fällen, in denen eine Vermehrung der Mastzellen der Haut konstatiert worden ist, keine Vermebrung der Mastzellen des Blutes vorlıanden ist. Die einzige Krankheit, bei welcher man eine Vermehrung der Mastzellen des Blutes findet, ist die myelogene Leukämie. So kann es denn anch kein Wunder nehmen, da 3 man speziell in der dermatologischen Literatur die Mastzellen meist als histiogenen Ursprungs auffaßt. Insbesondere ist es Unna, der diesen Standpunkt mit Sclıärfe vertritt.

1) Vortrag, gehalten in Verein für innere Medizin in Berlin, an 16. Oktober 1903 2) Anmerkung bei der Korrektur. Auf die Arbeit Leo Ehrlichs (Virchows Archiv 1904, Bd. 175, Heft 2), der eine Genese der Plasmazellen aus hypertrophischen Bindegewebszellen annimmt, kann hier nicht mehr eingegangen werden.
Unna betont die histiogene Entstehung der Mastzellen. Histopathologie S. 187 spricht er von einer Auswanderung von Leukozyten aus den Gefäßen und von Mastzellen aus der Kutis in das Epithel, stellt also beide Prozesse einander gegenüber. Bekanntlich hat auch Un a zuerst auf die starke Vermehrung der Mastzellen bei der Urticaria pigmentosa hingewiesen. "Sie sind aber nicht," sagt Unna, wie ich noch im Jahre 1887 glaubte, hier als Wanderzellen zusammengeflossen, sondern an Ort und Stelle. wie aucl seitherige Erfahrungen gelehrt, entstanden, und zwar einfacb aus den daselbst befindlichen Bindegewe bszellen durch Aufnahme der Mastzellenkörnung. “ An einer anderen Stelle sagt er: "An den Blutgefäßen entlang zieht siclı aber ein förmlicher Belag mit Mastzellen bis zum Hypoderm hinunter. Bei der Besprechung des Vorkommens von Mastzellen im perikarzinomatösen Infiltrate gibt Unna die Gründe an, aus denen er eine Wanderung der Mastzellen annimmt. Er sagt: "Wir lernen daraus mit Sicherheit. dal3 ursprünglicl feste Elemente des Bindegewebes nach Aufnahme spezifischer körniger Bestandteile unter dem Einflusse einer besonderen Chemataxis beweglich werden können." W'eitere Angaben Unnas über das Vorkommen von Mastzellen bei verschiedenen Erkrankungen der Haut können hier iibergangen werden. Im Gegen. satz zu Unna stehen einige andere Autoren. A udry (Monatshefte fïr praktische Dermatologie 1896, Bd. 22), bemerkte in seiner ausführlichen Arbeit: Mit Vorliebe suchen die Mast\%ellen die Zone auf, welche die Lymphspalten und besonders die Blutgefäße begrenzen." "Bei ibrer Durchwanderung durch die Gefäßwandung selbst ist es mir " nicht grelungen. sie z.11 beobachten, doch halte ich mich für berechtigt, sie als Leukozyten zu betrachten." Schließlich nimmt aber A udry dieses Zugeständnis wieder lıalb zurück und meint, die Mastzellengranulation zejge einfach einen chemischen Zustand an, in den jede Zellart geraten kann. Er will also in den Mastzellen keine besondere Form von Zellen erkennen. A ildry erwähnt ïbrigens. dals auch Ranvier in den "Klasmatozyten" dieselben Gebilde beschrieben hat, die Ehrlich Mastzellen nannte. Diese Klasmatozyten sind Lymphozyten.

Delbanco streift kurz in einer Arbeit iiber Erysipeloid (Deutsche Jedizinal-Zeitung 1898, No. 78) Untersuchungen, die er nit Leistik ow iiber die Histopathologie des weichen Schankers angestellt. Die Forsclıer stieß̉en wiederlıolt innerhalb der Gefäße auf polynukleäre Leukozyten, deren Protoplasma Mastzellenkörnelung zeigte.

Eine besondere Stellung in der Frage nimmt Pappenheim ein (Virchows Archiv Bd. 166. Monatshefte für praktische Dermatologie 1903) In einem Brief an nich präzisiert Pap penheim seinen Standpunkt. Er untersclieidet zwei Atren von Mastzellen: 1. feingekörnte, mononukleäre, histiogene und 2. . grobgekörnte, polynulukleäre, hämatogeule. Die histiogenen Mastzellen sind aus Bindegewebszellen hervorgegangen; sie sind entweder fixe, "sessile“ Zellen odter von ersteren abstummende Wanderzellen. Beim "Frosch fanden sich Endothelien mit Mastzellengranulation, die in der Haut rorkomulenden Mastzellen sind beim Frosch anssclıließliclı histiogen. Bei Säureern rrelangen die listiogenen Mastzellen nicht in das Blut, wohl aber im Exsudate.

Ich persönlich habe bei einer systematischen Durcharbeitung woh] der gesainten Hautpathologie des Menschen nurl bei sehr ausgedehnten Stıdien der mir zugänglichen Tierhautkrankheiten miclı viel mit der Mast. zellenfrage zu beschaaftigen Gelegenheit gelıabt, ohne an der Meinung irre zu werdell, daß die Mastzellen der Haut histiogenen Crsprung:s seien, also Abkümmlinge der Bindegewebszellen darstellen. A uffallend war allerdings die Tatsache, dals man Kernteilungen oder sonstige Teilungsvorgänge an den Mastzellen trotz der anlberordentlich grol3en Vermelırung bei verschiedenen Hatutkrankleiten nicht hat feststellen können. Da wurde ich durch zufällige Cntersuchung eines Präparates aus der Tierhantpathologie ron dieser meiner bislierizen Ansiclit abgebracht. Im Zoologischen Garten wurden zwei tote liaarlose Ratten gefunden, von denen mir eine durch die Liebenswïrdigkeit des Herrı Dr Tornier rom Zoologischen Museum übergebell wurde. Die nähere Untersucbung ergab, dals die Haarlosigkeit der Ratte nicht etwa auf dem Ausfall der Haare aus irgend einer äulßeren oder inneren Krank. heitsursaclie bernhte, sondern durch eine mangelhafte Anlage der Haare orler eine Rückbildıng der eventuell vorher angelegten Haare, also durch eine Hypotrichosis zu erklären war.

Auf die feinere Anatomie der recht komplizierten Hautkrankheit werde ich an anderer Stelle eingehen. Hier sei nur bemerkt, daß das Bild beherrscht wird von zystenartigen Bildungen, in denen sich z.11sammengesetzte konzentriscl gelagerte Hornmembranen befanden. E handelt sich um erweiterte Haarbälge, in denen noch Teile des äußjeren und der inneren Wurzelscheiden vorhanden sind. Ich nehme wenigstens nach Analogie an daß die Hornmassen ron der inneren Wurzelscheide abstammen. Die Gründe dafür werden an anderer Stelle auseinandergesetzt werden. Zwischen den Haarzysten finden sich Rund zellenansammlungen in geringer Zahl und Gröbe, sie bilden keinen wesentlichen Zug im Krankheitsbilde. Wenn die Haarzysten obelflächlich liegen, stellen sie meerbusenartige Einbuchtungen der Haut dar; sie enthalten teilweise noch die Haarscheidenhornbildung. In den tieferen Partien der Haut sind die Haarzysten oval; nicht selten sieht man Reste der Haarsclıäfte mit starker pathologischer Verhornung de 
inneren W'urzelscheide; zuweilen beobachtet man auch Querschnitte normaler Haare. Die Gefäße erscheinen durchgängig stark gefüllt und dilatiert; periarterielle Infiltrate sind nicht häufig.

Meine Aufmerksamkeit wurde durch eine eigentümliche Lagerung der Mastzellen in den tiefsten unmittelbar der Faszie aufliegenden Partien erregt. Zur Färbung benutzte ich die von Un $\mathrm{n}$ a angegebene Methode (Monatshefte für praktische Dermatologie 1894, Bd. 19, S. 367): 1. Fürbung 5 Minuten in polychromem Methylenblau (Grübler), Auswaschen in Wasser. 2. 1/4 Minute in Glyzerinäthermischung (Grübler) oder einige Minuten in bis zur Portweinfärbung verdünnter Glyzerinäthermischung; Auswaschen in Wasser. 3. Alkohol, Xylol, Balsam Mastzellengranulationen werden metachromatisch rot, Kerne blau. Vorhärtung in Alkohol oder 5-10\% Formalin gibt gleich gute Resultate.

Eine ganze Reihe von Präparaten errab folgendes Bild: in einer deutlich dem Verlauf präformierter Hohlräume folgenden Linie liegen zum Beispiel 12 Mastzellen hintereinander. Es handelte sich nicht etwa um eine Kette, sondern die länglichen Zellen lagen ohne jeden bestimmten Abstand in kürzeren oder größeren Zwischenräumen in dem Hohlraum, dem geschlïngelten Verlauf desselben sich anpassend. Der Beweis der Mastzellennatur wurde durch die Metachromie (Rotviolettfärbung bei Tinktion mit polychromem Methylenblau und Differenzierung mit Glyzerinäther) einerseits, andererseits durch die Form der Zellkörper gregreben. Die Zellen sind meist länglich, zejgen keinen scharf gefärbten Kern; dus ganze Protoplasma ist mit kleinen tief rotviolett gefärbten Granulationen besetzt. Ein Teil der Granulationskörperchen liegt abgesprengt neben den Zellen.

Es ervibt nun eine genaue Untersuchung, daß die langen Hohlräume, zu denen die Mastzellenketten in Beziehung stehen, Kapillaren sind. $\mathrm{Da}$, wo noch im Schnitte rote Blutkörper nachweisbar sind, ist es leicht, die Kapillaren als Blutkapillaren anzusprechen; wo dieser Nachweis nicht gelingt, muls die Frage offen bleiben, ob Lymphkapillaren oder blutleere Blutkapillaren vorliegen. Die Beziehung der Mastzellen zu den Gefälen ist besonders dann gut zu erkennen, wenn eine Mastzelle sich halb. quer zur Längssichtung der Kapillare gelegt hat. Da der Längsdurchmesser der Mastzelle größer als der Querschnitt der Kapillare ist, scheint sich die dünne Kapillarwand um die Mastzelle gevissermaßen auszubuchten.

Dasselbe Bild wiederholt sich sehr häufig in den einzelnen Präparaten. Bald findet man im Verlauf einer langen (das heißt lange im Schnitt befindlichen Kapillare) 12-14 dicke Mastzellen in gewissen Abständen hintereinander, bald sieht man im Verlauf eines kürzeren Kapillarstücks nur 4-5 Mastzellen. Zuweilen erblickt man sogar acht sehr große Mastzellen eng aneinander gedrängt, wachsenden Sproßpilzen gleichend. Nur in dem der Muskelfaszie unmittelbar aufliegenden Abschnitt der Haut ist das Thänomen eigentlich deutlich und anffälig. In dem oberen. von den

Haarzysten durchsetzten Teil der Haut finden sich auch zahlreiche Mastzellen; die eigentümliche Lagerung ist aber entweder nicht

nachweisbar, oder aber erst bei speziell auf die Frage gerichteter Aufmerksamkeit mehr oder weniger deutlich.

Form der Mastzellen. Die Mastzellen erscheinen entweder als länglich $30: 6 \mu$ große Gebilde oder als rundlich, beziehungsweise polygonale 13:13 große Bildungen, die in der Mitte eine Lücke für den ungefärbten oder wenig gefärbten Kern zeigen. Es finden sich auch ovale an einem oder beiden Enden zugespitzte Formen. Auch über eine seitliche Fläche gekrümmte nierenförmige Gebilde kommen vor $10-15 \mu$ im Längsdurchmesser, 8-12 $\mu$ im Querdurchmesser dürften die Maße der größten Zahl von Zellen sein.

Lage der Mastzellen zu den der Endothelien. In einer großen Zahl von Kapillaren liegen die Mastzellen so dicht der Wand an, daß sich zunächst die Ueberzeugung dem Untersucher aufdrängt, die Endothelien haben sich in Mastzellen umgewandelt. Diese Auf- fassung stößt allerdings schon a priori auf schwerwiegende Bedenken. Wir wissen, daß die Mastzellen Wanderzellen sind; die Annahme, da $B$ die Endothelien nach Aufsaugung der basophilen Granulationen aus der Blutflüssigkeit durch die Membrana propria der Gefäße hindurch wandern und ein endothelloses Gefäß zurücklassen sollten, widerstrebt unseren Erfahrungen. Bei genauer Durchforschung der Präparate findet man aber sehr viele Stellen, an denen man innerhalb des Ge. fäßes die dicke blaurot gefärbte granulierte Mastzelle unmittelbar neben der länglichen, feinen hellblau gefärbten Endothelzelle sieht. Besonders deutlich ist in größeren Hautgefäßchen die Trennung der Endothelien von den Mastzellen.

Extrakapilläre Lagerung der Mastzellen. Selbstverständlịch kann man das Hindurchtreten der Mastzellen durch die Gefäß. wände nicht rerfolgen; immer wenn man eine halb in der Kapillare liegende Mastzelle sieht, ist der Einwand zu machen, daß die Zelle vielleicht teilweise in einer andern Ebene liegt, als die Kapillare, was sie ja auch bei einer Wanderung schließlich tut. Immerhin kann man alle Stadien zwischen der intrakapillären und extrakapillären Lagerung verfolgen, w'enn man die oben geschilderte Lagerung der Zellen als Beweis ihrer Lage in den Gefäßen gelten läßt. So sieht man z. B. in der Mitte eines ganz mastzellenfreien Gesichtsfeldes einen Längs schnitt eines Gefäßchens, das prall mit roten Blutkörperchen angefüllt ist. Zwei Mastzellen liegen anscheinend auswandernd auf der Gefäßwand. andere liegen in verschiedenen Abständen von dem Gefäß. An einer andern Stelle durchläuft ein bogenförmig gekrümmtes Gefä $\beta$ das Gesichtsfeld. Deutlich unterscheidet man in dem Gefäß die blaugefärbten Leukozyten von den ungefärbten, gelb erscheinenden Erythrozyten. Eine runde Mastzelle liegt anscheinend intravasal neben den deutlich sichtbaren, blaugefärbten, länglichen Endothelien, eine andere längliche Mastzelle liegt bereits extravasal.

Genügen nun aber die geschilderten Bilder, um eine einfache intrakapilläre Lagerung der Mastzellen anzunehmen? Schwerwiegende Bedenken stellen sich dieser Auffassung entgegen. Obwohl bei schwacher Vergrößerung völlig der Eindruck erweckt wird, daß die genau den Kapillaren folgenden Zellketten innerhalb der GefäBwände liegen, zeigt sich doch bei ganz genauer Einstellung und starker Vergrößerung, daß kleine Niveauunterschiede zwischen den Erythrozyten, den Epithelien, den Leukozyten und den Mastzellen vorhanden sind. Freilich kann man sich leicht überzeugen, daß auch, was ja a priori zu erwarten ist, die in den Kapillaren rorhandenen Blutkörper nicht in einer Ebene liegen. Da die Mastzellen um ein mehrfaches größer sind, als die Leukozyten, ist die scheinbare Lagerung in verschiedenen Ebenen ohne weiteres verständlich. Dieser Umstand verhinderte auch, daß absolute Klarheit durch die Herstellung allerfeinster Schnitte bereitungsweise durch Zupfpräparate gewonnen wurde. Ich möcht bemerken, daß es mir bei dem in Formalin gehärteten Präparat nicht gelang, feinere Schnitte als $3 \mu$ (d. h. Mikrotomteilstriche) herzustellen.

Bei Berücksichtigung aller dieser Momente bin ich aber doch zu der Ueberzeugung gekommen, daB die Mastzellen nicht in den Gefäßen, sondern auf den Gefäßwandungen liegen, vielleicht auch zum Teil in die Gefäße hineinragen. Gegen ein Zirkuliereı der Mastzellen in den Gefäßen, speziell in den Kapillaren, spricht ihre Größe.

Es war schon oben betont, daß zuweilen die Mastzellen lănger sind, als die Kapillaren breit sind, und deshalb sich querzustellen genötigt werden. Eine solche Querstellung innerhalb der Kapillare würde aber den Flüssigkeitsstrom hemmen, was wieder durch rückläufige Stauung zum Ausdruck kommen müßte. Eine Querstellung auf der Gefäßwand ist aber ohne Schwierigkeit zu verstehen.

Gibt man die Richtigkeit meiner Beobachtung zu, so ergibt sich notwendig zur Erklärung der Forgänge eine Hy pothese, die meines Erachtens nach geeignet ist, die Erscheinungen wenigstens verständlich zu machen.

A us dem Blute wandern Leukozyten, oder wahrscheinlicher, Lymphozyten aus, welche in der Gefäßwand liegen bleiben (Stomata) un hier entweder direkt aus der Blutflüssigkeit die basophilen Granula anziehen oder, was wahrscheinlicher ist, nur die chemischen Stoffe in sich aufnehmen, welche ihnen die Produktion der Mastzellenkörnchen ermöglichen. Nachdem die Mastzellen in der Gefäßwand eine Art Reifung durchgemacht haben, wandern sie aus und dringen schließlich in alle Schichten der Haut ein. Die ron vielen Autoren schon beschriebene Vorliebe der Mastzellen für die Lagerung in den Lymph scheiden der Gefäße erklärt sich so ohne Schwierigkeit. Bei der von mir untersuchten Tierhautkrankheit lagen die Verhältnisse für die Erkennung des Vorgangs vielleicht besonders günstig; es handelt sich wohl um eine kongenitale Krankheit oder wenigstens um eine kongenitale Krankheitsanlage, die zu einer erheblichen Störung im Gefăßsystem mit konsekutiver Stauung geführt hat.

Ich bin mir bewuBt, eine Hypothese gegeben zu haben, die noch vielfach der Stützen, insbesondere durch weitere bestatigende Befunde bedarf. Da aber diese hämatologischen Fragen auBerhalb des mich in absehbarer Zeit beschäftigenden Gebietes liegen, hielt ich mich zur 
Publikation meiner Befunde. so wenig abgeschlossen sie auch sein mögen. für berechtigt.

Ueber die weiteren Untersuchungen kann ich kurz berichten. Schon a priori war es nicht wahrscheinlich. daß der Vermehrung der Mastzellen in der Haut eine Vermehrung der Mastzellen im Blute parallel gehe. Ist doch in der menschlichen Pathologie eine Zunahme der Blutmastzellen. wie bei chronischen Entzündungen der Haut, festgestellt worden. In den Quer- und Längsschnitten der großen Blutgefäße der Haut findet man sehr selten einige Zellen. die durch die charakteristische Granulation an Blutmastzellen erinnern; sehr deutlich sind diese Bilder bei der für Blutuntersuchungen ganz unzweckmäßigen Betrachtung des in den Gefäßen konservierten Blutes nicht. Etwas häufiger sieht man typische längliche „Gewebsmastzellen". Wieder aber zeigt die leichte Niveaudifferenz. daß es sich um Vlastzellen. die wahrscheinlich auf der Gefäßwand liegen. handelt. Leider war es nicht möglich das Blut der Ratte in der üblichen Weise im fixierten Deckglaspräparat auf Mastzellen zu untersuchen. Das Tier war in toto (nach Eröffnung der Brusthöhle und Bauchhöhle durch ganz kleine Schnitte) in 5\% Formalin konserviert worden. Die Cntersuchung des Herzblutes ergab daher keine brauchbaren Präparate. Aus demselben Grunde möchte ich auch der Untersuchung der Milz keinen besonderen Wert beilegen. Ich fand sehr zahlreiche einkernige Zelleı. die neutrophile Granulationen (bei Färbung nach May-Grünwald) zeigten. und auch viele Zellen (einkernige Lymphozyten). deren Granulation bei Mastzellenfärbung schwach rötlich-violett gefärbt war. Die Furbung unterschied sich sehr wesentlich von einer gelungenen Mastzellentinktion. Irgend welche Schlüsse kann ich aus diesen Befunden nicht ziehen. weil ausgedehnte Kontrolluntersuclungen. z. B. iiber den Einfluß der Härtung auf die Farbenreaktionen erforderlich widren.

Schließlich erwähne ich. daß ich die Haut und das Blıt normaler Ritten untersucht habe. sowie die von L. Micliaelis angegebene Metbode der Färbung der Mastzellen unter Vermeidung der Anwendung von Wasser bei der haarlosen Ratte angewendet habe, ohne irgend welche bemerkenswerten Befunde zu erlieben: ich fand zwar Mastzellen, die so gelagert waren. daß eine Bezielsung zu den Ka. pillaren angenommen werden konnte. Bilder jedoch. die so einwandsfrei waren, wie bei der geschilderten Hautaffektion. habe ich nicht gefunden. 